\title{
A challenge to scientific integrity: a critique of the critics of the GMO rat study conducted by Gilles-Eric Séralini et al. (2012)
}

\author{
Ulrich E. Loening
}

\begin{abstract}
This paper reviews the many criticisms of the publication by Seralini et al (2012) which has led to so much controversy, was retracted and then republished in this journal. Seralini et al found that a GM maize and its associated herbicide Roundup resulted in numerous chronic abnormalities in rats. The vehemence of the critics is not matched by their evidence; it is often based on entrenched assumptions and on mis-representing published material. The arguments have challenged normal healthy scientific dialogue, and appear to be driven by other motives. A further interpretation of Seralini et al's results on tumour formation is suggested. The probability that Seralini et al's results are significant is sufficient to justify further study.
\end{abstract}

\section{Background}

Genetically modified organisms (GMOs) in agriculture have been controversial from the start. The debate frequently reaches beyond scientific/technical issues and demonstrates the poor state of scientific dialogue and public understanding [1]. The arguments have again become acute following the publication by Seralini et al. [2] which reported that a herbicide resistant GM maize and the associated herbicide Roundup each led to longterm chronic disease in rats including early formation of tumours. This paper was a challenge to GM interests and provoked immediate and widespread criticism and press coverage [3]. The speed of response was such that one could question whether this was disinterested scientific exchange [4]. The editor of the journal finally retracted the paper because, after much discussion, it was considered insufficiently definitive: "Ultimately, the results presented (while not incorrect) are inconclusive...." [5]. Inconclusive results are not usually sufficient reason for retraction. Inconclusiveness would indeed apply to the initial publications of many or even most of the great discoveries in biology, as the author of [4] has pointed out. The controversies continue.

Correspondence: uel@ednet.co.uk

Department of Zoology, and Director of the Centre for Human Ecology,

University of Edinburgh, Edinburgh, Scotland, UK
The Seralini et al. paper was republished in this journal [6] with responses by Seralini to the critics; however, the critics' publications have not been retracted and remained in the historical record. Heinemann [4] defended Seralini et al.'s paper long before it was retracted, and Meyer and Hillbeck [7] demonstrated how the criticism by the European Food Safety Authority (EFSA) suffered from double standards. Following these, I question here whether there exists sufficient substantive evidence given by critics (other than EFSA) to deny the validity of Seralini et al.'s findings. I refer here to the original Seralini et al. (2012) article and not to its republication, which has been edited with changed figure and table numbers.

The criticism of Seralini et al. was so vehement in condemnation that it threatened (and ultimately prevented) normal scientific discourse. I examine the validity of the critical arguments and the assumptions behind them and how the conduct of this controversy reflects on the integrity of science. In the process, I suggest some further interpretations of the Seralini et al. paper. There is a documented history of antagonism towards papers that demonstrate negative effects of GM crops [8]. It is important to study the rationale behind such antagonism.

\section{Springer}

(c) 2015 Loening. This is an Open Access article distributed under the terms of the Creative Commons Attribution License (http://creativecommons.org/licenses/by/4.0), which permits unrestricted use, distribution, and reproduction in any medium, provided the original work is properly credited. 
Outline of the Seralini et al. (2012) paper

Seralini et al.'s experimental design followed closely that of earlier work by others, with which it is compared in Table 1 of their paper. The major purpose was to extend the trials from the accepted protocol of 90 days to over 700 days, because some evidence of disease was already apparent after only 90 days.

In outline, Sprague Dawley (SD) rats, 8 weeks old, were randomly assigned into 10 groups of 10 for each sex (200 animals in total) and fed with rat chow supplemented with three proportions of the GM maize NK603, grown with or without Roundup (R). Control groups were fed with rat chow supplemented with the non-transgenic maize as control. The drinking water for three further groups was supplemented with three concentrations of Roundup, the lowest being below the permitted standards for drinking water, and a control. The rats were monitored twice weekly throughout, and blood samples were taken 11 times during the 750 days of the experiment. Body weight and food consumption were monitored but not reported. The health of these rats through life is described in figures, tables and photographs. An important difference from previous trials, and especially from Monsanto's trial given in their application for approval (released by the European Food Safety Authority in 2013), was the latter's use of some non-isogenic maize in control feeds. When the experimental rats showed effects that were within the spread of these controls, it was "concluded that this difference was not biologically meaningful." In this way, small effects up to 90 days were ignored.

To the authors' surprise many treated rats, especially females, developed tumours after a year, while the controls developed fewer tumours and later. The numerous effects on liver, kidney and pituitary functions were expected from the authors' previous data.

Sources of the criticisms of the Seralini et al. paper The widespread condemnation of the Seralini et al. paper was publicised via three routes:

1. The Science Media Centre was quick to publicise many comments online [9]. Publication was so fast that some critics had very little time to study the paper adequately;

2. About 17 letters to the editor of Food and Chemical Toxicology (FCT) criticised the paper and asked for its retraction [10];

3. A fuller paper of criticism appeared later (Arjo et al. [11]), and an informative report was published by The Flemish Interuniversity Institute for Biotechnology (Vlaams Interuniversitair Instituut voor Biotechnologie) (VIB) [12], (quoted by [13] in the submission to FCT); the European Food Safety Authority compiled similar materials [14] which were the subject of critique by Meyer and Hillbeck [7]. Seralini et al. reply to other parts of Arjo et al.'s arguments, in their republished paper in this journal [6].

\section{Main Text}

\section{General criticisms}

Most comments included general accusations about the quality of the work, such as:

"The study appeared to sweep aside all known benchmarks of scientific good practice and, more importantly, to ignore the minimal standards of scientific and ethical conduct..." (Arjo et al. [11]), and

"Throughout their manuscript, Séralini et al. ignore clear indications that there is something fundamentally wrong in their experimental design" (Grunewald [13]).

Such judgments were widespread. Does the evidence justify them?

Many challenged the study design for not following OECD or EPA protocols for safety testing for tumours. The experiments, however, were not safety tests. A safety test requires a large enough trial not to miss a rare occurrence. No one would be satisfied by a safety test with only 10 animals. These particular OECD and EPA protocols are therefore not relevant. It must also be stressed that differences in experimental protocol do not necessarily imply that one or another is flawed, as Meyer and Hillbeck [7] pointed out.

Table 1 The ratios of tumour days in GMO-treated female rats to controls over different time periods, taken from Seralini et al.'s Figure 2 by summing numbers of tumours appearing over 25-day periods. A ratio of 1 would indicate no difference from control; this is approached in the last 100 days, showing numerically what can be seen in the figure, at which time the experimental findings become less significant, as described in the text

\begin{tabular}{lll}
\hline Days & Ratio tumour days treated/control & Number of tumours $\times 25$-day periods \\
\hline 100 to 550 & 3.04 & 91 \\
100 to 650 & 2.38 & 179 \\
550 to 650 & 1.92 & 88 \\
650 to 750 & 1.23 & 94 \\
\hline
\end{tabular}


Arjo et al. identify the basis of poor design thus: "The key flaw in the paper is the poor study design, which is based on the discredited hypothesis that inserting a gene into the genome of a crop species is inherently more likely to produce unintended, unexpected and hazardous characteristics than would be the case using conventional breeding."

There are several different fundamental errors in this statement, which assumes that an inserted gene can have no adverse action other than the intended action. This is a commonly held assumption and is related to the concept of "substantial equivalence", but it is factually wrong; the hypothesis in the quote is not discredited by the majority of scientists. This is considered more fully in the "Discussion" section.

\section{A multiplicity of errors}

The critics point out a diversity of errors within the Seralini et al. paper. The VIB report concludes with a bullet-pointed list of identified errors including the following:

"There is no mention of how the nutritional balance was kept in the various diets. If you replace 22 or $33 \%$ of the food with maize, you change the percentage or the amount of carbohydrates, protein, fats, fiber, vitamins and so on, in the diet."

"There is no indication whatsoever of whether the genetically modified maize originating from the land that was sprayed with Roundup contained traces of the degradation products of Roundup and, if so, how much there was, and how this compared to the amount of Roundup that was fed to all the animals".

Arjo et al. listed "many major critical errors" in their Table 3. For example, under the heading "False or unsubstantiated statements", they and others give several instances of inadequate reporting of results and of tests that were made. They claim that there is insufficient detail about the feed materials; as in the VIB quote above, and by Wager [15]:

"a robust experiment would also include a random, unrelated diet, e.g., one derived from organic maize)..... Critical details on how much food was consumed by each rat are absent, making it impossible to establish any dose/response relationship."

These comments fail to appreciate that all diets, including the controls, were equally balanced with $33 \%$ maize (GM or not); sources and details of the feed materials are described by Seralini et al., but more detail would have been useful. The GM maize grown with Roundup was as in usual agricultural practice, and maize was tested for contaminating pesticides. It is correct that total feed intake and rat weights were not reported, but they were monitored. Again, more data would be useful.

A common complaint was that neither the concentration and stability of glyphosate in the drinking water nor the amounts consumed by rats were given: Roundup was given ad lib at stated concentrations. Since ad lib provision represents a realistic regime, as it would be for farm animals and for humans, the amounts consumed by rats are not relevant. The use of Roundup is considered separately below.

Many complained that "Control data [was] not always included in the limited cases where data are presented to support the conclusions". With few exceptions, this is incorrect. All tables and figures other than the photographs of rats with tumours (Figure 3, Seralini et al.) include the relevant control data; (note that the important Figure 2 is ignored by all, as described below).

The above are typical examples of criticism that arises from misreading or misunderstanding the paper and selection of mostly minor or irrelevant matters. In many cases, further information would indeed have enriched the paper and might have been provided in a supplementary paper online.

I conclude that these critical comments could have been addressed in a workshop presentation of the results and most complaints could be answered by provision of more data. None are sufficient, however, to invalidate the data and results of Seralini et al.

Most critics comment on the number of deaths in control and experimental groups, for example pointing out that male rats that were given Roundup at the highest concentration actually lived a little longer than the controls. All of these comments, although quoting from Seralini et al.'s Figure 1 which gives the time course of occurrence of deaths, deal mainly with the totals of deaths at the end of the experiment. This, notably, is the fault in Arjo et al.'s Table 1 [11] which by not mentioning the timing is a misrepresentation. Similarly, the VIB paper [12] quotes only the final end-of-experiment histograms from Figure 1, omitting the timing. While it does seem extraordinary that at the highest doses of Roundup male rats live a few days longer than controls, no significance can be attached to such small differences. (It is just conceivable that the highest concentration of Roundup offers some protection, in a non-monotonic manner; see [16].) Since Seralini et al. themselves draw no essential conclusions from Figure 1, the matter does not justify further concern.

\section{The incidence of tumours}

Figure 2 in Seralini et al. shows that treated rats acquired tumours earlier than did the controls. It was 
Figure 2 with its accompanying photographs of tumours that became the subject of widespread concern, media coverage and controversy. The two most frequent and substantive criticisms of the paper were that the numbers of rats were too small with too few controls and that Sprague Dawley (SD) rats suffer spontaneous tumours anyway, making the conclusions of Seralini et al. meaningless. EFSA, for example, states: [14] “....the observed frequency of tumours is influenced by the natural occurence of tumours typical of this strain, regardless of any treatment. This is neither taken into account nor discussed in the Séralini et al. (2012) publication."

Yet most of the critics in the several communications cited above never mention Seralini et al.'s Figure 2 and do not discuss or criticise its findings. One critic who did mention Figure 2 [17] complained: "There are other confusing sentences that also reflect poor editorial work, for example "Up to 14 months, no animals in the control groups showed any signs of tumors whilst 10-30\% of treated females per group developed tumors, with the exception of one group (33 \% GMO + R)."....I was left wondering if anyone had really read the paper carefully." Careful reading shows that the quoted sentence is factually correct. Another also found the paper difficult to interpret [18] and questioned whether control rats got tumours because of the absence of photographs of control tumours. This critic concluded: "Numerically, we cannot tell, because they are absent also from Figure 2." Every section of Figure 2, however, shows a control curve. The critic's error could be a misreading, perhaps the result of the too rapid reply required by the Science Media Centre (the same critic also misread Table 2, which included the 10 controls, not fewer; the wording here is clear but can be misread) VIB, [12] without mention or quote from Figure 2 concludes: "Only once you have increased the size of the groups significantly will the chances that you have divided the animals incorrectly drop considerably. This is the second fundamental flaw in the research design used by Séralini et al. They use far too few animals per treated group."Their criticism is considered further below under "Statistical issues" section.

Since Figure 2 is of core importance for the whole paper, I offer my interpretation as follows:

Simple inspection of Figure 2 shows that the curves of appearance of tumours in treated rats rise ahead of those representing the control tumours. Incidence of tumours in control rats are generally consistent with the published literature (such as the five references quoted by Arjo et al., see below) and serve to check against unforeseen local anomalies. It is important to note that there is considerable variation in the literature about the incidence of tumours in control SD rats. Many studies concluding that GM materials are safe have used such variable historical controls. The variation serves to increase the background "noise" and this tends to hide small indications of disease, as reviewed in [19] and explored by Meyer and Hillbeck [7].

As rats age and their pathology increases, so the difference between treated and control rats decreases; in other words, the signal-to-noise ratio of experimental to control reduces. Yet most critics considered only the incidence of tumours at the end of the experiments, that is, when the signal-to-noise ratio must have become low. For example, 1 of the 17 critics in [10] showed ingeniously that the figures for tumour incidence in Seralini et al.'s Table 2 looked like random numbers. The figures he quoted, however, were also end-of-experiment data. Nevertheless, the total of pathologies in the treated animals remained always greater than in the controls even this Table 2. Critics (including VIB) fail to discuss the timing of appearance of tumours in Figure 2. It is the timing of appearance of tumours that, to me, is significant and should not be overlooked.

In Figure 2, the tumour incidence in treated and control rats can be seen as the areas enclosed between the respective curves and the $\mathrm{x}$-axis for any chosen time period. The units for these areas can be defined as "accumulated tumour days". These can be easily summed from the graphs in Figure 2 in order to put tangible figures to otherwise intuitive visualisation. One can then express the results as the ratio of "tumour days" in treated rats to "tumour days" in control rats for any specified period. For example, I added the tumour days in Figure 2 "FEMALES GMO" (top right) by summing together the tumours as they appeared under all three feeding doses of 11,22, and $33 \%$ GMO (divided by 3 to apply per 10 rats) neglecting any differences between doses. In this way, 30 treated rats are compared to 10 controls. The ratios of tumour days of treated to control rats are shown in Table 1.

The change in the ratio with time provides a measure of internal control. In this example, during the period of 100-650 days, there are nearly $2 \frac{1}{2}$ times as many tumour days in the treated rats as in the control rats, while in the last 100 of these (550-650 days), the ratio is only 1.92 . This suggests that significant results are limited to about 550 days. No statistical analysis could deny that the large ratio of 3.04 up to 550 days is significant.

However, results presented in this way could be subjected to serious errors at the early stages when very few tumours occur. For this reason, the total numbers of tumour days are indicated. (see also the VIB criticism under the "Statistical issues" section below.)

One can also express the results as time of appearance of the first five tumours per 10 rats (i.e., $50 \%$ occurrence). This is 710 days for controls, 470 to 630 for GMO treated and 470 to 530 for Roundup treated. This provides a convenient way to summarise the findings, akin to an "LD50". 
The above analysis is consistent with the authors' conclusions that the treated rats developed tumours significantly sooner than did the control rats. The results are also consistent with the critics' arguments and with the literature, which shows that SD rats get tumours anyway, but only later in life.

I conclude that, contrary to the criticisms, SD rats are the appropriate animal for these experiments precisely because of their susceptibility to late tumorogenesis, in agreement with Meyer and Hillbeck [7]. This idea is taken further in the "Discussion" section.

In summary, Figure 2, which with its accompanying photographs gave rise to the most publicity (yet has been the most ignored by all the critics), acts as the crucial and informative data about the onset of tumours. The figure shows clearly that there is an effect by a GM crop as well as by its associated herbicide. There is no reason to extrapolate these findings, however, beyond the experimental results. The conclusions of Seralini et al. need not apply to other GM systems, as seems to have been assumed by many anti-GMO interests, as well as by most of the critics.

\section{Statistical issues}

Among the many criticisms about the statistics used, VIB elegantly describe the possible errors from using too few controls: "The chances that we will have made groups of 10 among the females in which in the one instance two and in the other instance nine animals will spontaneously develop tumors, or four and eight instead of six, are extremely good" "This is a fundamental error in the research design: there are too few control groups in relation to the treated groups."

It is of course possible that of the 10 groups of 20 rats (10 for each sex) tested, 1 group has a lower incidence of spontaneous tumours than the rest. Such a group would be an outlier in the distribution. An outlier like this could happen to be chosen by the researchers as a control. The probability of a whole group of 10 rats showing a lower incidence of tumours is low, and there is a 1 in 10 probability that such a group would be chosen as control. In this unlikely case, Seralini et al.'s conclusions would indeed be rendered invalid. This argument seems to answer: "...how, in such a long-term experiment, can you differentiate between tumors that occur spontaneously from those that occur as a consequence of eating genetically modified maize, or drinking Roundup."

The analysis I give probably answers the above by considering the time course of appearance of tumours. Neglect of the timing of tumour appearance was a universal error of the critics.
Seralini et al.'s Figure 5 shows the SEMs of the numerous parameters measured at 15 months, (450 days) arranged in order from the highest increase to the highest decrease of a parameter, over control values. It would be difficult to argue that these are without significance. Any experimental biologist, searching for effects and finding results such as Seralini et al. did in Figures 2 and 5, would assume that they had found something sufficiently meaningful to justify further experimentation. Imagine that if the experiments had given the opposite results and showed that the onset of tumours was delayed by the treatments, the same critics may have (sceptically) accepted those findings as hints towards finding ways to delay human tumourogenesis!

More animals and statistical analysis might have allowed one to determine a greater or lesser degree of confidence; but certainty was not claimed by Seralini et al. and nor was it required, since theirs was not a test for safety. The results in the (largely ignored) Figure 2 leave us with the likelihood that the effects are real and the probability that they are of significance.

Scientific common sense must be the initial way to assess this experimental data; statistical analysis follows when needed, but usually it is better to repeat the experiment with appropriate improvements. In this case, with hindsight, two sets of 10 control rats would have enhanced the evidence. Meanwhile, Seralini et al.'s results remain significant, even if the level of confidence is below $95 \%$.

\section{Roundup and glyphosate}

There was much criticism that glyphosate concentrations and levels of its consumption were not given by the researchers and that only a whole formulation of Roundup was described and used. Arjo et al. took this further suggesting that Seralini et al. "justify the use of commercial formulations rather than the pure active ingredient on the tenuous basis that environmental exposure is to the whole product. The weakness of this argument is apparent from the differing behavior of formulations and their ingredients in terms of environmental stability, mobility in ground water, ..." [11].

Roundup is the commercial material being tested; one cannot know a priori whether any toxicity is due to glyphosate or an adjuvant or to their combined actions. This is a common error that assumes that because glyphosate is the active herbicide principle it is also the material to be tested for other activities; this puts assumption before experiment. There are many reports about the toxicity of glyphosate itself (e.g., $[19,20]$ and of its untoward effects on the microbial community in soils [21]. 
Many others assume that there should be a doseresponse relationship, for example: "...highest incidence of tumors supposedly found in the animals administered the lowest dose. These conclusions are not only implausible, but they are entirely discordant with the body of literature already available for glyphosate." [11]. However, there is a growing body of literature about damage caused by glyphosate, such as [20] quoted above; also, non-monotonic dose-response curves are common in biology [16].

I conclude that the criticisms about the use of Roundup by Seralini et al. were not relevant to the experiment, which was concerned with the material used in agricultural practice, not with glyphosate itself.

\section{The 90-day protocol}

The conventional 90-day test period has become the norm, and most deny the need for longer term experiments: Arjo et al. defend the 90-day protocol thus: "This particular rat strain exhibits a $45-80 \%$ incidence of spontaneous tumors in the absence of any exogenous factor, depending on the diet and whether or not fed ad libitum (Prejean et al. 1973; Davis et al. 1956; Keenan et al. 1996; Suzuki et al. 1979; Thompson et al. 1961). The rats normally begin developing these spontaneous tumors after 90 days, and are used in shorter-term experiments to ascertain tumorigenicity,...."

None of the above references given in the Arjo et al. paper anywhere mention 90 days, and none of them give a time scale for the appearance of tumours. Each states, however, that rats develop tumours only in older age, such as "540 days" (Davis et al. and Prejean et al.) or older than "18 months" (Suzucki et al.) and, with a complex chow formulation, "in female rats ranging in age from 11 to 30 months (21.78 average)" (Thompson et al.); Keenan et al. report tumour incidence later in life, reduced under a restricted diet, a similar finding to the older one of Davis.

One has to conclude that the above quoted paragraph is erroneous reporting of all five references. The case for longer term experiments remains. One might note that the protocol of 90 days became the norm after it was set arbitrarily by Monsanto in its applications and has been since adopted by others without question or any apparent justification.

\section{Conclusions from the critical evidence}

The criticisms described above contain several serious flaws and omissions, such as the following:
- Misreporting (e.g., of the five references and their association with 90-day trials);

- Misunderstanding (e.g., the omission of Figure 2 and the distortion of the use of Roundup);

- Unwarranted assumptions (e.g., putting presumption before experiment, such as expecting a doseresponse effect and the discrediting a common hypothesis (see below);

- Misreading of data, especially regarding experimental controls (e.g., complaints that there were none in critical places).

The criticisms also included useful comments, such as the dearth of detailed information on food consumption and rats' weights; these were measured by Seralini et al. but not reported and could have been provided online as supplementary information.

However, as illustrated with the examples given, I could not find any substantive material definitive enough to invalidate the paper. The most substantial and repeated criticisms were either false or could be answered easily. We are left, predominantly, with polemical criticisms that fail scientifically.

This is an unsatisfactory situation for the interests of biotechnologists in gene transfer (GMOs) as well as for any deeper understanding of the issues among biologists and the public. It is worthwhile, therefore, to examine underlying modes of thought that led to such vehement denial.

\section{Discussion}

However, improbable they may seem, Seralini et al.'s results indicate that the GM system has caused disease in the rats and that the causal agent(s) is not a macro change in the feed but a micro unknown non-visible change. Such a change would escape notice in analyses that search for unintended changes in metabolic materials, as described by Herman and Price [22] in their plea that: "suspect unintended compositional effects that could be caused by genetic modification have not materialized on the basis of this substantial literature. Hence, compositional equivalence studies uniquely required for GM crops may no longer be justified on the basis of scientific uncertainty".

If, then, the crucial changes in the GM crop are micro and more like hormonal ones as Seralini et al. suggest, it also follows that the experiment can be a difficult one to confirm depending, as it must, on unknown small effects.

\section{What underlies the criticisms of Seralini et al.?}

Arjo et al. claim (as quoted above) that the hypothesis of damage from inserted genes is discredited. The evidence for this claim is clear: that the methods of gene transfer are a success, that the crop plant does have the characteristics intended, and that GM crops are grown and 
consumed around the world with no obvious adverse properties. This also supports the basic principles of genetics on which the GM technology is based. Arjo et al.'s claim seems to be shared implicitly by most or all of the critics of the Seralini et al. paper. It seems, however, that problems begin to appear only when searches for other and unintended effects become more thorough and extended, as in Seralini et al.'s paper. There is now growing evidence that the claim is false, with the following implications:

1. Arjo et al.'s accusation of "bad design" due to the "discredited" hypothesis is false, because the hypothesis that inserted genes may produce unwanted effects is a finding, not a presumption. That the accusation is false is also underlined by the internationally binding legislation of the UN Cartagena Protocol on Biosafety of 2000 carried forward in 2014 and representing the minimum consensus of over 160 signatory nations.

2. Of all the assertions made this one most requires supporting references despite its widespread acceptance, but none are given nor are they in similar criticisms by other critics, except for the repeated references to other long-term trials that showed no harmful effects.

3. Many studies show that inserted genes do exert multiple effects, reviewed in [23]. The basics of gene structure and function lead one to expect this. Thus, a) most genes are pleiotropic, exerting unknown diversities of functions; b) many proteins themselves have multiple activities, including control functions; c) most "junk" DNA is transcribed (as emphasised by ENCODE), and the RNA has complex multiple control functions; d) as a result, a randomly inserted gene is likely to hit some function; e) the transferred gene, with a strong promoter added and introns removed, may have activities other than those intended. (Much of this was apparent 14 years ago [1].)

4. Therefore, the idea that inserted genes exert no unintended damaging effects is an implicit hypothesis which has become an assumption and is now entrenched as a consensus. This consensus cannot be applied as criticism of Seralini et al.'s paper merely because it indicated the opposite of those many other results.

5. This consensus also allows the continued misuse of the ill-defined term "GMO", leading for example, to the frequent claims that "Genetic Modification" is merely a form of speeded up breeding carried out ever since agriculture began. Maybe this is intended to allay public fears by showing how the process is ordinary, occurs in nature and is nothing new, (gene modification is after all the basis of evolution).
Thereby, the new and exciting developments of gene transfer methods become buried from view, rather than being extolled. Public understanding of science is therefore betrayed and this stance becomes counter-productive.

There are so many such instances of accepted philosophies or dogmatic assumptions in the history of science that the geneticist Waddington, who introduced the idea of epigenetics, coined the acronym COWDUNG (COnventional Wisdom of the DominaNt GroUp), [24]. Those who have worked on or tried to publish findings that run contrary to a prevailing consensus have been condemned or ignored and suffered years of opposition, even if proved to be ultimately correct (for example, recall Peter Mitchell's long struggle to combine the (at the time almost opposed) fields of structural cell biology of membranes with the biochemistry of soluble enzymes to create his chemiosmotic hypothesis, now the accepted mechanism of how mitochondria and chloroplasts work; or the years of disbelief in Howard Temin's evidence for "reverse" transcription because at the time the Central Dogma of molecular biology was simplistically held to mean that information can flow only one way from DNA to RNA; even now we still call them "retroviruses"). These two examples are of healthy (if over-severe) scepticism which resisted the challenges to conventional perceptions. In these cases, increasing evidence over time led to new understanding.

But scientists who have published provocative results about GM crops have been vilified beyond any scientific justification [8], as seems to be the case here. Seralini et al. challenged the dominant consensus. Following this case, Carrasco [20] was also attacked, even beyond his death. In contrast to my two examples above, such attitudes of vilification undermine and ultimately prevent scientific progress.

\section{The rat tumours}

Seralini et al.'s results deserve closer scrutiny. There appears to be a subtle influence of diet, which is already hinted at in other literature as cited above. One can compare the situation with that in humans, who are similarly prone to tumours with increasing age and in whom tumourogenesis is certainly hastened by known and unknown influences (as indicated by the incidences of cancer in different countries). For humans, no one denies the need for long-term studies; the rat 90-day trial could be compared with a tobacco smoking trial in human teenagers which is stopped in their 20s, long before lung cancer becomes apparent.

As many critics have pointed out, however, after more than 10 years of GMO crops, there are no signs of disease in animals or humans that can be ascribed to 
GM technology. Seralini's answer to this is obvious: that farm animals do not live long enough [25]; to which one can add that humans live too long and that there has not been time enough for major problems to develop. There is, however, evidence that some harm is becoming apparent. Among these is the opening paragraph of Keenan et al.'s paper (quoted by Arjo et al. in connection with the 90-day test, see above) which points out that "Laboratory rat survival in 2-year carcinogenicity studies has been declining over the past three decades." This is re-iterated later [26]. Similarly, a farming report [27] lamented the increase in developmental abnormalities and abortion in cattle, threatening the industry over three decades. The report blamed oestrogen-like pollution. There have been many anecdotal reports from several countries of new abnormalities appearing on farms in recent years, especially following the introduction of Roundup for weedmanagement. Teratogenic effects of Roundup have been reported in vertebrates [20] and reviewed in [19]. Early reports predate the use of GM crops, but Roundup and other herbicides have a longer history of widespread use.

Thompson et al.'s (1961) experiments (quoted in Arjo et al.) involved complex diets which could be the reason for the large temporal spread of onset of tumours (11-30 months). Perhaps they are a presage to Seralini et al. indicating that some features of rat chow can lead to abnormalities in sensitive animals. It should be noted that when tumour development is referred to as "spontaneous" this denotes ignorance of cause rather than lack of cause. One may therefore be able to find conditions in which SD rats develop fewer tumours than in normal laboratory controls. Given the probability that Seralini et al.'s findings have some validity, it would be worthwhile to try an "inverted" repeat of their experiments, in which improved diets are compared to "normal" controls, such as a trial with $\mathrm{SD}$ rats kept under semiwild conditions on an organic farm. The UK Government's National Health Service recommendation to consume "Five a Day" of fruit and vegetables is an uncontrolled trial of this sort, with one of the assumed benefits being a reduced incidence of cancer (although this is not at all proven).

\section{Conclusion}

\section{The scientific/technological attitude}

How is it that many distinguished gene transfer scientists have condemned Seralini et al. so vehemently on such a weak basis? The simplest answer is that theirs was such a bad paper but then why the vehemence? I have to conclude that long and deep engagement in the science of gene transfer (usually but incorrectly named "GMO", as explained above) and safety testing has resulted in a blindly accepted consensus among a circle of gene engineering interests that makes any contradictory findings unacceptable. One is justified in asking as in [4] whether the criticism was motivated by something other than science, some conscious or unconscious imperative to defend the current COWDUNG (which is supported, one may note, by large corporate interests as also are the deniers of humancaused climate warming). A lack of consensus among scientists in general about the safety of GMOs was published in this journal while this paper was being written [28].

Had the criticisms been worthy of rigorous scientific dialogue, useful progress could have resulted. For example, if one can identify a difference between insertion of a DNA cassette and insertions due to recombination in meiosis or insertions of transposons, then one might discover new features about the mechanisms and control of gene action. But the effect of the criticisms was to "silence" the discussion and to inhibit the scientific enquiry that Seralini et al. had initiated.

If a GM crop threatens a potential problem, then the response should be to check that problem and then fix it. Responsible further study might lead to new discoveries. As it is a far from resolving antagonism towards GMOs, the critics have fostered mistrust among both the public and among many biologists. Their efforts will be counter-productive for the further development of the subject. The matter has now become so polarised that it threatens the rationality of further debate, just at a time when gene transfer methods are becoming more sophisticated.

One can now question whether and how the present gene transfer technologies should be further applied to agriculture. Thus, Arjo et al. write (perhaps surprisingly): "New findings that affect entrenched opinions and economic interests have their detractors, particularly those with a potential global impact. One of the key ethical principles of science states that "not all that can be done must be done" (Institut Borja de Bioètica 2012). Science itself is neutral, but individual scientists and their supporters inevitably have particular interests...." [29] Precisely so, the great successes of applied molecular biology have led to their own shared and selfreinforced certainties, a COWDUNG or "Pie in the Sky" [30]. Much of modern science has indeed been applied on the basis of "I can, therefore I do" [31]. The "plurality of opinion" about GMOs may be due to the differing attitudes about scientific progress and its applications. A re-think is in order. Seralini et al. [25] note that their paper was "a first step in the iterative investigation of the long-term health effects on mammals of these commercial products that should be replicated independently, as 
well as on developing mammals". Their paper has to stand or fall through further experiment over time, but the criticisms reported above can only hinder progress and remain an indictment of the quality of the underlying scientific discourse.

\section{Competing interests}

The author declares that he/she has no competing interests.

\section{Acknowledgements}

I thank Dr Michael Antoniou for encouraging me to write and bringing the Arjo et al. paper to my notice and Prof Jack Heinemann for detailed critique of an early draft, also Daisy Martinez for critical reviewing of the manuscript, and all three referees of the paper for useful suggestions, including the critical one who unwittingly confirmed my thesis. They and many other colleagues have provided essential references and I thank them for the valuable discussion.

Received: 6 November 2014 Accepted: 11 June 2015

Published online: 21 June 2015

\section{References}

1. Loening UE The Science missing from the GMO debate. 1999; [http://web.archive.org/web/20010412085302/http://www.che.ac.uk/People/ Ulrich/missingscience.htm] accessed 5th March 2015.

2. Séralini G-E, Clair E, Mesnage R, Gress S, Defarge N, Malatesta M, et al. Long term toxicity of a Roundup herbicide and a Roundup-tolerant genetically modified maize. Food Chem Toxicol. 2012;50:4221-31.

3. Butler D. Rat study sparks GM furore. Nature. 2012;489(484):474. see also Editorial.

4. Heinemann JA. Letter to the editor. Food Chem Toxicol. 2013;53:442.

5. Retraction notice. Food and Chemical Toxicology 2014; 63: 244

6. Séralini et al. Republished study: long-term toxicity of a Roundup herbicide and a Roundup-tolerant genetically modified maize. Environmental Sciences Europe 2014; 14-31.

7. Meyer H, Hillbeck A. Rat feeding studies with genetically modified maize-a comparative evaluation of applied methods and risk assessment standards. Environ Sci Eur. 2013;25:33.

8. Waltz E. Battlefield - papers suggesting that biotech crops might harm the environment attract a hail of abuse from other scientists. Nature. 2009;461:27-32.

9. [http://www.sciencemediacentre.org/expert-reaction-to-gm-maize-causingtumours-in-rats/] Accessed 5 March 2015

10. About 17 papers in Food and Chemical Toxicology 2013; 53:440-475.

11. Arjo G, Portero M, Pin ${ }^{\sim}$ Ol C, Viñas J, Matias-Guiu X, Capell T, et al. Plurality of opinion, scientific discourse and pseudoscience: an in depth analysis of the Seralini et al. study claiming that RoundupTM Ready corn or the herbicide RoundupTM cause cancer in rats. Transgen Res. 2013;22:255-67.

12. VIB. 2012; [http://www.vib.be/en/news/Documents/ 20121008_EN_Analyse\%20rattenstudie\%20S\%C3\%A9ralini\%20et\%20al.pdf] Accessed 5 March 2015

13. Grunewald W, Bury J. Comment on "Long term toxicity of a Roundup herbicide and a Roundup-tolerant genetically modified maize" by Séralini et al. Food Chem Toxicol. 2013;53:447-8.

14. European Food Safety Authority. Review of the Séralini et al. (2012) publication on a 2-year rodent feeding study with glyphosate formulations and GM maize NK603 as published online on 19 September 2012 in Food and Chemical Toxicology. EFSA J. 2012;10(10):2910.

15. Wager R. Letter to the editor. Food Chem Toxicol. 2013;53:455-6.

16. Vandenberg LN, Colborn T, Hayes TB, Heindel JJ, Jacobs DR, Lee D-H, et al. Hormones and endocrine-disrupting chemicals: low-dose effects and nonmonotonic dose responses. Endocr Rev. 2012;33:378-455.

17. Langridge P. Letter to the editor. Food Chem Toxicol. 2013;53:441.

18. Malony, M, 2012; [http://www.sciencemediacentre.org/expert-reaction-togm-maize-causing-tumours-in-rats/] Accessed 5 March 2015

19. Antoniou M, Habib MEM, Howard CV, Jennings RC., Leifert C, Nodari RO, Robinson CJ, Fagan J. Teratogenic effects of glyphosate-based herbicides: divergence of regulatory decisions from scientific evidence. J Environ Anal Toxicol. 2012; S: 4-17.
20. Paganelli A, Gnazzo V, Acosta H, López SL, Carrasco AE. Glyphosate-based herbicides produce teratogenic effects on vertebrates by impairing retinoic acid signaling. Chem Res Toxicol. 2010;23:1586-95.

21. Kremer RJ, Means NE. Glyphosate and glyphosate-resistant crop interactions with rhizosphere microorganisms. Eur J Agronomy. 2009;31:153-61.

22. Herman R, Price W. Unintended compositional changes in genetically modified (GM) crops: 20 years in research. J Agric Food Chem. 2013;61:11695-701.

23. Wilson AK, Latham JR, Steinbrecher RA. Transformation-induced mutations in transgenic plants: analysis and biosafety implications. Biotechnol Genet Eng Rev. 2006;23:209-37.

24. Waddington, CH. Tools for thought. Paladin 1977 Frogmore, St Albans Herts, AL2 2NF.

25. Séralini G-E Mesnage R, Defarge N, Gress S, Hennequin D, Clair E, Malatesta $\mathrm{M}$, et al. Answers to critics: why there is a long term toxicity due to a Roundup tolerant genetically modified maize and to a Roundup herbicide. Food Chem Toxicol. 2013:53:461-8.

26. Hubert M-F, Laroque P, Gillet J-P, Keenan KP. The effects of diet, ad libitum feeding, and moderate and severe dietary restriction on body weight, survival, clinical pathology parameters, and cause of death in control Sprague-Dawley Rats. Toxicol Sci. 2000;58:195.

27. Science news online, April 2002 Unexplained abortion and abnormal ageing in cattle. Veterinary Sciences Tomorrow, Universiteit Utrecht. http://vetscite.org/2002/04/unexplained-abortion-and-abnormal-ageing-incattle/ April 2002.

28. Hilbeck A, Binimelis R et al. No scientific consensus on GMO safety. Environmental Sciences Europe 2015 [http://dx.doi.org/10.1186/s12302-014-0034-1]

29. Institut Borja de Bioètica Francesc Abel i la Biotètica, un llegat per a la vida, 1st edn. Editorial Proteus. 2012 (quoted from Arjo et al)

30. Simmonds NW. Pie in the Sky. Tropical Agriculture Association Newsletter 1997; June: 1-5.

31. Loening U E, 2003, I can, therefore I do. [http://www.thetimes.co.uk/tto/ opinion/letters/article2075878.ece] Accessed 5 March 2015

\section{Submit your manuscript to a SpringerOpen ${ }^{\circ}$ journal and benefit from:}

- Convenient online submission

- Rigorous peer review

- Immediate publication on acceptance

- Open access: articles freely available online

- High visibility within the field

- Retaining the copyright to your article

Submit your next manuscript at $\boldsymbol{\wedge}$ springeropen.com 